CARNETS DE Carnets de géographes

GÉOGRAPHES.

$6 \mid 2013$

Géographie des faits religieux

Sacrée nature, paysages du sacré

Géographie d'une nature réenchantée?

Etienne Grésillon et Bertrand Sajaloli

(2) OpenEdition

Journals

Édition électronique

URL : http://journals.openedition.org/cdg/932

DOI : $10.4000 /$ cdg. 932

ISSN : 2107-7266

Éditeur

UMR 245 - CESSMA

Référence électronique

Etienne Grésillon et Bertrand Sajaloli, «Sacrée nature, paysages du sacré », Carnets de géographes [En ligne], 6 | 2013, mis en ligne le 01 septembre 2013, consulté le 23 septembre 2020. URL : http:// journals.openedition.org/cdg/932 ; DOI : https://doi.org/10.4000/cdg.932

Ce document a été généré automatiquement le 23 septembre 2020.

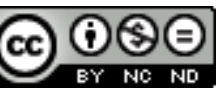

La revue Carnets de géographes est mise à disposition selon les termes de la Licence Creative Commons Attribution - Pas d'Utilisation Commerciale - Pas de Modification 4.0 International. 


\title{
Sacrée nature, paysages du sacré
}

Géographie d'une nature réenchantée?

\author{
Etienne Grésillon et Bertrand Sajaloli
}

1 Le colloque «Sacrée nature, paysages du sacré » tenu à Orléans du 22 au 24 janvier 2009, fût l'occasion d'aborder les faits religieux et leurs incidences sur les représentations et la gestion des milieux naturels. Révélant les ressorts du sacré dans les perceptions de la nature et dans les paysages à différentes périodes (antiquité, médiévale, moderne et contemporaine), les chercheurs traquent, sur différents territoires (Europe, Amérique du nord et du sud, Afrique, Extrême-Orient, MoyenOrient), les manifestations du sacré dans les pratiques et représentations, en abordant aussi bien les croyances polythéistes antiques (grecques, romaines et égyptiennes), animistes que les religions du Livre (judaïque, islamique, chrétiennes). Cosmopolite et pluri-temporel, les travaux dressent ainsi un tableau vivant et diversifié, sur le rapport entre religion et paysage qui ont nourri les deux ouvrages à paraître fin 2013 aux Presses Universitaires Panthéon Sorbonne sous la direction de B. Sajaloli et E. Grésillon : Le sacre de la nature et Par bois, monts et marais. De l'ici-bas à l'au-delà.

Dans ce carnet de lecture, il s'agit de présenter les soubassements théoriques du projet et de montrer la portée de ce travail pour l'analyse des paysages. L'objectif dans un premier temps est de montrer que nous assistons dans les sociétés occidentales à un enchantement ou un ré enchantement du monde (Gauchet, 1985 ; Moscovici, 2002) qui sacralise la nature et les paysages associés. Pour analyser le sacré, il s'agit selon Pierre Deffontaine (1966) que le géographe ne se contente pas de " noter les répercussions géographiques des faits de religion sur le paysage, car il réduirait ainsi le point de vue religieux à des éléments extérieurs et physionomiques, laissant délibérément de côté le domaine majeur de la vie intérieure " (Deffontaines, 1966, p. 1718). Nous désignerons par sacré la recherche ou la confrontation de l'homme avec une présence suprahumaine qui implique un rapport non-matériel à l'espace. Dans un deuxième temps, pour comprendre ce réinvestissement idéel sur la nature, nous présenterons la manière dont la géographie et les géographes ont intégré les relations entre les faits religieux et les espaces naturels. Et enfin, à partir des différents travaux présentés dans les deux 
ouvrages, nous esquisserons une synthèse sur les différents ressorts du sacré dans les paysages naturels.

\section{Un sacre de la nature partagé ?}

3 Les éco-guerriers de Fontainebleau et d'ailleurs, luttant contre une gestion perçue comme productiviste, invoquent dans leur Déclaration commune, « des droits sacrés et inaliénables de la terre et des espèces" «afin que tous les acteurs de la vie ne se laissent jamais opprimer ou avilir par la tyrannie, afin qu'ils conservent les bases de leur liberté et de leur pérennité » (Eco-guerrier, 2013). La nature est sacralisée, et son sacre légitime leur combat.

Dans un registre plus mesuré, les Journées des plantes, les fêtes dédiées à un légume (fête du potiron, de la courge, de la châtaigne...), à un arbre (fête du chêne, du hêtre, du sapin, de l'olivier), à un fruit (fête de la cerise, de la pomme, de la poire, de l'abricot...) attirent chaque année des centaines de milliers de visiteurs alors que les festivals des jardins, comme celui de Chaumont-sur Loire, connaissent un engouement grandissant. Domestiquée, idéalisée, la nature déplace les hommes pour des dévotions postmodernes. Sauvage, ou du moins faiblement influencée par l'homme, elle compose alors de " véritables sanctuaires " pour reprendre une expression fréquemment utilisée par les écologues. La nature est sacralisée, et son sacre justifie sa protection.

5 Sur un plan plus intime et personnel, et peut être en réponse aux crises du monde contemporain, le désir de «communier » avec la nature, celui plus diffus d'en faire encore partie, expliquent en partie la réussite de l'alimentation biologique, de la phytothérapie, des onguents et soins naturels et des pratiques de plus en plus répandues de loisirs verts. La nature sauvage ne doit donc pas être sauvée pour elle seule, mais aussi pour l'homme (de Miller, 2007 ; Terrasson, 2008) car c'est dans la mesure où les individus prennent conscience de leur intériorité qu'ils sont conduits à comprendre les richesses naturelles autour d'eux. Près de la nature, l'homo urbanicus recherche un équilibre perdu, une harmonie. Objet d'une quête spirituelle, la nature bienfaitrice, réparatrice, est dotée de pouvoirs supra-humains.

6 Ainsi, on assiste à un sacre de la nature qui, partagé par des acteurs sociopolitiques, doit créer du lien social comme par exemple dans les jardins partagés qui fleurissent dans les villes (Mairie de Marseille, 2013), à l'apparition d'une nouvelle foi, qui transcende tous les clivages culturels et politiques, d'une quête du moins, personnelle, collective, en réponse aux peurs du moment. En témoigne la floraison d'ouvrages destinés au grand public traquant dans les espèces floristiques ou faunistiques un symbole religieux (Bilimoff, 2006 ; Cocagnac, 2006 ; Dumas, 2000 ; Impelluso, 2004). Par l'emploi récurent d'images religieuses (déluge, paradis, apocalypse), la place du sacré dans les représentations sur la nature semble partagée. Pourtant, ce sacre de la nature n'en est pas moins ambigu, complexe voire instrumentalisé.

7 D'une part, il succède assez brutalement à une longue phase dans laquelle l'exploitation des ressources naturelles, la «maîtrise» de la nature par l'homme, alimentaient le paradigme du progrès. Ce sacre est donc récent, contemporain des crises -sociales, économiques, environnementales- dénoncées dans le rapport Brutland (Jollivet, 2001 ; Larrère, 1997 ; Ramade, 1999) et doit être lu à l'aune de l'avènement du développement durable (Dalage, 2008). Dès lors, il s'agit de l'inscrire dans l'histoire des rapports homme-nature (Beck, 2006 ; Robic, 1992), ce qui suscite une tension entre le sacré qui, 
par essence, dès lors que l'on est croyant, n'est pas un construit social et l'approche historique, attentive aux échelles de temps et d'espace, qui confronte évolutions des croyances et mutations des structures politiques, économiques, technologiques, culturelles et sociales.

8 D'autre part, il accompagne la rationalisation de nos sociétés occidentales fondée sur les sciences et les techniques et organisée selon l'économie libérale, dans lesquelles, l'indice, le taux (CAC, Dow Jones, IDH, PIB...) étalonnent le monde. Le chiffre jouxte ainsi le goupillon. Mais cette rationalité conquérante, fidèle compagne de la mondialisation, n'est pas dénuée de spirituel, ou, n'est pas sans favoriser en réaction, son regain (Bourg, 2010). Il s'agit donc d'inscrire ce sacre de la nature dans une deuxième tension entre objectivité et subjectivité, entre science et spiritualité (Latour, 2002), particularisme culturel et modèle planétaire. Le recours à la science des paysages, notamment dans une approche post-moderne (Deleuze et Guattari, 1980), fournit une piste de remédiation scientifique. Les empreintes paysagères du sacré, d'autant plus masquées qu'elles marquent des milieux dits naturels, c'est-à-dire à la fois régis par seul le divin ou par des processus écologiques, agissent comme autant de signes de l'appropriation sociale du spirituel.

9 Enfin, s'il est très répandu, ce sacre est très loin d'être partagé, pensé et pratiqué de la même manière. Concept valise, englobant, il pose de véritables difficultés épistémologiques et théologiques à la fois aux laïcs qui dénoncent ou se gaussent d'un fétichisme vert et aux religieux qui se méfient d'un retour de l'animisme. Significatif est ainsi le débat sur le désenchantement ou le réenchantement de la nature (Gauchet, op. cit. ; Moscovici, op. cit.) qui reflète une troisième tension entre immanence et transcendance, entre sacré et sainteté, profane et religieux. Tension religieuse mais aussi politique dans la mesure où les Institutions (religieuses, laïques, nationales, internationales, gouvernementales ou non...) s'emparent du sacré pour cautionner leurs actions, étayer leur influence et leurs pouvoirs. D'où des accusations croisées de totalitarisme et de profanation, de récupération et d'instrumentalisation (Ferry, 1992; Deléage, 1993) sur fond de conflits quant aux choix à effectuer pour la gestion environnementale de notre planète (Latour, 1999; Brunel, Pitte, 2010). Le sacre de la nature appartient donc au politique (Raffestin, 1985) et n'échappe pas à des recompositions dont la déesse Gaïa, ou le Christ vert (Bastaire, 2009), constituent quelques figures de proue (Lovelock, 2006).

\section{Le sacré et la géographie, un long interdit épistémologique}

10 Le fait religieux a longtemps été négligé par les géographes : avant les années 1980, le seul ouvrage français de géographie traitant spécifiquement du thème, celui de Pierre Deffontaines a été publié en 1948. Les actes du Festival International de Géographie de Saint-Dié de 2002, dont le thème était "Religion et géographie ", explicitent ce manque d'intérêt par le triomphe d'une géographie [humaine] d'inspiration rationaliste pour qui le religieux sentait quelque peu le soufre. Les géographes abandonnèrent donc le thème aux sociologues, aux historiens, aux anthropologues et aux civilisationnistes, quelques rares géographes s'associant à ces derniers dans les équipes du CNRS " (Actes du Festival de SaintDié, 2013). Ce à quoi s'ajoute aussi une posture sociale d'aménageur, de science 
appliquée, qui réfute donc l'indicible et s'appuie (se réfugie ?) sur la réalité du terrain, le regard davantage fixé au sol que vers les cieux.

11 L'intégration du sacré dans une pensée scientifique soulève de redoutables questions épistémologiques et personnelles que certains auteurs ont affrontées (Caillois, 1950 ; Castelli, 1974 ; Dardel, 1990 ; Girard, 1972 ; Otto, 2001 ; Piveteau, 1999 ; Racine, 1999). Les années 1980 et 1990 signent le renouveau de la géographie culturelle en France qui annonce un développement des études sur les faits religieux. Les numéros consacrés à ce thème par la revue Géographie et Cultures, dirigée par Paul Claval, illustrent bien cette renaissance : de 1992 à 2002, 16 des 40 premiers numéros de cette revue (soit au total une trentaine d'articles) évoquent ces questions (Claval, 1992 ; Pitte, 1992 ; Mercier, 1997). De même, la revue Hérodote, animée par Yves Lacoste, entre 1983 et 1998, aborde la question religieuse dans 12 numéros distincts sur les 90 publiés. Enfin, une vingtaine d'ouvrages et autant d'articles (issus d'autres revues de géographie que celles signalées supra) croisant identité religieuse et territoire paraissent dans ces deux mêmes décennies (Assayag et Tarabout, 1997; Bruneau, 1998; Racine, 2001 ; Rieucau J., 1998...). Mais dans tous les cas, les thèmes retenus sont soit directement liés à la localisation des religions, y compris à la grande échelle des territoires (Bertrand et Muller, 1999), soit ouvertement géopolitiques, notamment pour les Proche et Moyen Orient (Hérodote 1984 ; 1985), soit plus civilisationnels quand l'Asie ou l'Amérique latine prévalent. De même, cette géographie religieuse, souvent assortie d'atlas, décrit volontiers les lieux sacrés anthropisés (monastères, cimetières...), les migrations et les impacts socio-économiques liés aux pratiques religieuses comme les pèlerinages (Debarbieux, 1993 ; Dumortier, 2002 ; Dupront, 1996). Citons, l'ouvrage de Jean Bernard Racine qui, interrogeant sa propre foi au prisme de son engagement scientifique, dévoile la dimension spatiale de cette pratique sociale qu'est la foi des hommes (Racine, 1993)

12 Ainsi, la question du rapport entre le paysage et le sacré n'est-elle, du moins en Europe occidentale, guère abordée. Citons les travaux de Giuliana Andreotti qui, sortant de la stricte inscription des pratiques rituelles dans l'espace, amorce une réflexion sur les liens entre paysage et religion (Andreotti 1997). Etienne Grésillon dans sa thèse : Une géographie de l'au-delà? Les jardins de religieux catholiques, des interfaces entre profane et sacré effectue un travail heuristique sur le lien entre spiritualités catholiques et paysages dans des jardins appartenant à des communautés religieuses en France (Grésillon E. 2009). Signalons également le numéro spécial de la revue Zones Humides Infos intitulé «Sacrées zones humides!» qui propose en 14 brèves présentations rassemblées par B. Sajaloli (Sajaloli, 2006) quelques éléments de réflexion sur les liens entre croyances et aménagement des lieux d'eau ou encore les travaux portant sur la perception des zones humides dans la Bible et son influence sur l'aménagement de l'espace (Sajaloli, Grésillon, 2013).

13 Ainsi, la géographie des phénomènes religieux, et plus généralement celle du sacré et du symbolique, est en plein essor. En atteste notamment, la participation d'une trentaine de géographes aux deux ouvrages mentionnés plus haut. Ce renouveau nourrit ainsi la démarche disciplinaire, la géographie, même physique, étant plus attentive aux influences des perceptions et des représentations sur les dynamiques de l'espace. 


\section{Une géographie des ressorts du sacré}

14 Le sacré renvoie à un objet géographiquement situé et apparaît comme socialement produit : il associe une expérience individuelle ou collective à un objet géographique. L'association se construit autour d'une perception du monde partagée ou, personnelle. Cette expérience n'est pas donnée de l'extérieur (Dardel, 1990) «dans une perception interprétée par l'intellect, mais rencontrée dans une expérience primitive : réponse de la réalité géographique à une imagination créatrice qui, d'instinct, cherche quelque chose comme une substance terrestre ou qui, s'y heurtant, l'irréalise en symboles, en mouvements, en prolongements, en profondeur» (Dardel, op. cit.: 19-20).

15 À partir des travaux des chercheurs ayant participés au colloque, nous cherchons ici à repérer les circonstances géographiques, historiques ou eschatologiques à l'origine de la sacralisation d'un lieu de nature, en associant les paysages à l'expérience sacrale des individus. Il s'agit de montrer comment les paysages naturels participent à la sacralisation du monde. La beauté, la vie, la mort, l'immensité des paysages ainsi que récits mémoriels rattachés à un espace contribuent fortement aux sentiments sacrés. Selon nous, ils en constituent les ressorts tangibles.

16 La beauté d'un paysage ou d'un objet est le premier ressort du sacré. Elisée Reclus dans l'Histoire d'une montagne exprime le plaisir « de satisfaire complètement » son regard « à la vue de ce que neiges, rochers, forêts et pâturages » offraient « de beau » (Reclus, 2011 : 28). Il ajoute : « je planais à mi-hauteur, entre les deux zones de la terre et du ciel, et je me sentais libre sans être isolé » (Reclus, op. cit.). Cette beauté lui procure un "doux sentiment de paix» (Reclus, op. cit.) qui pénètre son cœur. Cette beauté transcendantale est un signe ou une empreinte d'un au-delà divinisé ou non qui semble être le grand ordonnanceur. Ce ressort du sacré s'associe plutôt à des paysages ouverts (montagne, mer) qu'à des paysages fermés (forêt) ou à des petits éléments paysagers (arbres, grotte).

Le deuxième détonateur du sacré se situe dans une rencontre avec le mystère de la vie. Echappant en grande partie au contrôle anthropique, la dynamique de la vie, libre, autonome, infiniment diverse, suscite un émerveillement qui renvoie à une intelligence, à un ordre supérieur qui prend une dimension supra-humaine. Ainsi, la nature offre de telles richesses, participe d'une telle complexité fonctionnelle, qu'elle révèle nécessairement une puissance supérieure (Grésillon, Arrif, 2012). Et ce, dans toutes ces dimensions écologiques : la végétation (arbre, forêt), la faune, les quatre éléments (eau, feu, terre, air). Les arbres, les forêts, les mares et les étangs procurent ainsi une expérience sacrée. La fertilité ainsi que le dynamisme de la nature aiguisent la sensibilité de certains individus qui voient dans les changements du paysage un signe ou une empreinte d'une entité supra-humaine.

18 À cette vie féconde s'opposent les abysses de la mort, cette fin éternelle que rien ne réveille. La manifestation du sacré surgit du questionnement sur la vie après la mort. Le monde des morts, le cimetière, les montagnes, les marais, les grottes, les rivières peuvent être tour à tour des lieux dans lesquels la mort rode et éveille une incertitude sacrale (Pitte, 1992). Ce ressort du sacré se matérialise ainsi à la fois dans les paysages fermés (forêt sombre), mais également dans les paysages à risques (marais, mer, montagne). 
19 L'immensité des paysages ou des formes paysagères est le quatrième détonateur du sacré. Face à des paysages grandioses, l'individu peut ressentir sa petitesse et l'étendue des choses. Cette puissance accordée à une puissance supra-humaine provoque des émotions qui mélangent la peur, l'effroi, le dépassement, la grandeur... L'homme dans un sentiment d'infériorité veut interagir avec cette force qui dépasse l'entendement. Les paysages majestueux (mers, montagnes et forêts) sont plus propices à l'expression de ce ressort du sacré (Grésillon, 2009).

Le sacré se rattache également aux récits mémoriels qui donnent corps à l'expérience divine. L'individu ou le groupe construisent autour d'un paysage une histoire évoquant un événement qui dépasse l'homme terrestre. Dès lors, la bible, la thora, le coran peuvent être vus comme autant de textes religieux qui racontent topologiquement l'expérience de dieu d'un groupe d'individu (Halbwachs, 2008). Ils explicitent les ressorts géographiques du sacré pour les personnes fréquentant Israël, la Palestine, l'Arabie Saoudite mais également pour les fidèles qui observent des paysages associés à ces textes. Les déserts, les oasis, les mers, les montagnes dévoilent tous une part des récits des religions du Livre et se trouvent ainsi être des paysages potentiellement sacralisables. Les paysages identitaires rattachés à un groupe avec des limites qualifiées (massif montagneux, forêt, mer) sont des territoires qui permettent le surgissement d'un sacré mémoriel.

21 En conclusion Daniel Vidal présentait le sacré comme un « concept-joker assez flottant, en même temps qu'impératif, pour ne pas se perdre dans la dissolution de la pensée même qu'il avait fécondée " (Vidal, D., 2006). Dans ces recherches, le sacré a été un support idoine pour qualifier les rapports entre les faits religieux et les paysages. La géographie, par le biais des paysages, apparait donc réconciliée avec la religion.

Ces travaux fournissent in fine des clefs pour comprendre le poids du sacré dans la construction des paysages de nature. Il démontre à l'envi que l'idée d'un cosmos vidé du sacré s'avère réductrice. Sur tous les continents, des récits et des pratiques continuent d'entretenir une fascination pour une entité supranaturelle. Partout le sacré se réinvente et de nouveaux paysages naturels surgissent. La nature est un temple où de vivants piliers émettent des paroles... moins confuses peut être.

\section{BIBLIOGRAPHIE}

Actes du Festival de Saint-Dié, 2013 [en ligne], http://archives-fig-st-die.cndp.fr/actes/ actes_2002/index.htm

ANDREOTTI G., (1997) « Aspects généraux entre paysage et religion », Géographie et Cultures, n 23, pp. 77-88

ASSAYAG J., TARABOUT G. (dir.) (1997) Altérité et identité. Islam et Christianisme en Inde, Editions de l'EHESS, collection Purushartha, Paris, 365 p.

BASTAIRE J. (2009) Pour un Christ vert, Paris, Salvator, 128 p. 
BECK C. LUGINBÜHL Y., MUXART T. (2006) Temps et espaces des crises de l'environnement, Paris, Quae, $410 \mathrm{p}$.

BERTRAND J.-R., Muller C. (ouvrage collectif), (1999) Religions et territoires, collection géographie Sociale, Paris : L'Harmattan, $291 \mathrm{p}$.

BILIMOFF, M. (2006) Les plantes, les hommes et les dieux, enquête sur les plantes messagères, Rennes, Ouest France, $127 \mathrm{p}$.

BOURG D., ROCHE P. (dir) (2010) Crise écologique, crise des valeurs? Défis pour l'anthropologie et la spiritualité, Genève, Labor et Fides, 333 p.

BRUNEAU M. (dir.) (1998) Les Grecs pontiques, diaspora, identité, territoires, Paris : CNRS Editions, $247 \mathrm{p}$.

BRUNEL S., PITTE J.-R. (dir), (2010) Le ciel ne nous tombera pas sur la tête: 15 grands scientifiques nous rassurent sur notre avenir, Paris, Lattès, 352 p.

CAILLOIS R. (1950) L'homme et le sacré, Paris, Folio essais, 250 p.,

CASTELLI, E. (dir.) (1974) Le sacré : études et recherches, actes du 14e Colloque international sur la thématique de la démythisation, Rome, Aubier, $492 \mathrm{p}$.

CLAVAL P. (1992) «Le thème de la religion dans les études géographiques ", Géographie et Cultures, $\mathrm{n}^{\circ}$ 2, pp. 123-129

COCAGNAC, M. (2006) Les symboles bibliques. Lexique théologique, Paris, Cerf, 453 p.

DALAGE, A., AMAT, J.-P., FREROT, A.-M., GUICHARD-ANGUIS S., JULIEN-LAFERRIERE B. WICHEREK, S. (2008) L'après développement durable. Espaces, nature, culture et qualité, Paris, Ellipses, $352 \mathrm{p}$.

DARDEL E. (1990) L'Homme et la Terre, Paris, Éditions du CTHS, 199 p.

DEBARBIEUX B. (1993) « Du haut lieu en général et du mont Blanc en particulier » L'Espace Géographique, $\mathrm{n}^{\circ} 1$, p. 5-13.

DEFFONTAINES P. (1948) Géographie et religions, Paris : Gallimard, 439 p.

DEFFONTAINES P. (1966) Géographie des religions : la marque géographique des religions, pp. 1717-1725, in Journaux A., Deffontaines P., Brunhes J., Delamarre M., (dir.), Géographie générale, Paris, Éditions Gallimard, 1883 p.

DELEAGE, J.-P. (1993) «L'écologie politique, humanisme de notre temps », Ecologie Politique, numéro spécial, $\mathrm{n}^{\circ} 5$, hiver, pp. 1-14.

DUMAS A. (2000) Les Plantes et leurs symboles, Paris, Editions du Chêne-hachette livre, 128 p., 2000.

DUMORTIER B. (2002) Atlas des Religions : croyances pratiques et territoires, Paris, Editions Autrement collection Atlas, $63 \mathrm{p}$.

DUPRONT A. (1996) Pèlerinages et lieux sacrés, Paris : Encyclopedia Universalis, pp. 791-804

Eco-guerrier (2013) [en ligne] http://terresacree.org/ecoguerr.htm

FERRY L. (1992) Le nouvel ordre écologique, Paris, Grasset, 275 p.

GAUCHET M. (1985) Le Désenchantement du monde. Une histoire politique de la religion, Gallimard, Paris, 303 p.

GIRARD R. (1972) La violence et le sacré, Paris, Librairie Hachette Littératures, 487 p. 
GRESILLON E. (2009) Une géographie de l'au-delà ? Les jardins de religieux catholiques, des interfaces entre profane et sacré, thèse Doctorat, Université Paris IV-Sorbonne, Paris, vol. 1, 378 p.

GRESILLON E., ARRIF T. (2012) « Du jardin d'Eden à la continuité naturelle : chemin d'un imaginaire de désenchantement? »Cahiers d'ADES, n8, pp. 133-145

HALBWACHS M. (2008) Topographie légendaire des Evangiles en terre sainte, Presses Universitaires de France, 3ème édition, 388 p.

Hérodote (1984) « Géopolitique des Islams », n³5, 176 p.

Hérodote (1985) «Géopolitique des Islams », n³6, 160 p.

IMPELLUSO L. (2004) La nature et ses symboles, Paris, Hazan, 384 p.

JOLLIVET M. (2001) Le développement durable, de l'utopie au concept. De nouveaux chantiers pour la recherche, Paris, Elsevier-Natures Sciences Sociétés, 288 p.

LARRERE C., LARRERE, R. (1997) La crise environnementale, INRA, 302 p.

LATOUR B. (1999) Politiques de la nature. Comment faire rentrer les sciences en démocratie?, Paris, la découverte, $382 \mathrm{p}$.

LOVELOCK J. (2008) La Revanche de Gaïa, Paris, J’ai Lu, coll. «J'ai Lu Essai » (no 8579), 256 p.

Mairie de Marseille (2013) [en ligne] http://www.marseille.fr/sitevdm/document?

id $=9682 \&$ id_attribute $=48$

MERCIER G. (1997) « Les espaces religieux », numéro spécial de la revue Géographie et cultures, $\mathrm{n}^{\circ} 23,144 \mathrm{p}$.

MILLER (de), (2007) Le besoin de nature sauvage, Jouvence, Saint Julien-en-Genevois, 155 p.

MOSCOVICI, S. (2006) Réenchanter la nature, entretien avec Pascal Dibie, La Tour d'Aigues, Editions de l'Aube, $66 \mathrm{p}$.

OTTO R. (2001) Le Sacré, Paris, Petite Bibliothèque Payot, traduction de l'allemand par Jundt A., $285 \mathrm{p}$.

PITTE J.R. (1992) « Espaces des morts, espaces de culture », Géographie et Cultures, n 4, 144 p.

PIVETEAU J.-L. (1999) Le géographe et la foi, p. 255-263, in Bertrand J.-R., Muller C., Religions et territoires, Paris, L’Harmattan, 292 p.

RACINE J.-B, (1993) La ville entre Dieu et les hommes, Genève, Economica, 349 p.

RACINE J.-B. (1999) Le scientifique et sa foi religieuse ? De l'ordre du monde à sa transformation, p. 27-66, in Bertrand J.-R., Muller C., Religions et territoires, Paris, L'Harmattan, 292 p.

RACINE J.-L. (dir.), (2001) La question identitaire en Asie du Sud Est, collection Purushartha, Paris : Editions de l'EHESS, 405 p.

RAFFESTIN C. (1985) « Religions, relations de pouvoir et géographie politique », Cahiers de Géographie du Québec, 29, 76, pp. 101-107.

RAMADE F. (1999) Le grand massacre. L'avenir des espèces vivantes, Hachette, 288 p.

RECLUS E. (2011) Histoire d'une montagne, Paris, Infolio, p. 209

RIEUCAU J. (1998) « Sociétés et identifications territoriales. Permanence des lieux, territorialités religieuses et festives sur le littoral du Golfe du Lion », Annales de Géographie, n 604, pp. 610-636 
ROBIC, M.-C. (dir) (1992) Du milieu à l'environnement : pratiques et représentations du rapport homme-nature depuis la Renaissance, Paris, Economica, 348 p.

SAJALOLI B., (2006) « Zones humides et diffusion du christianisme, le cas du Laonnois (Aisne)? », Zones Humides Infos, $\mathrm{n}^{\circ}$ 54, $24 \mathrm{p}$.

SAJALOLI B. GRESILLON E., 2013 « La terre était vague et vide, l'esprit de Dieu planait sur les eaux (Gn 1,2). Eaux chrétiennes et paysages contemporains, du symbole au modèle », p. 75-86, in SAJALOLI, Bertand et SERVAIN-COURANT, Sylvie, Zones humides et littérature, Paris, Collection Journées d'Etudes du Groupe d'Histoire des Zones Humides, 142 p., 2013.

TERRASSON, F. (2008) La civilisation anti-nature : on ne peut vivre en parenté avec la nature sans comprendre ce que nous sommes, Paris, Sang de la Terre, 293 p.

VIDAL D. (2006) « Michel Carrier, Penser le sacré. Les sciences humaines et l'invention du sacré », Archives de sciences sociales des religions, 136 [en ligne] http://assr.revues.org/index3889.html.

\section{INDEX}

Thèmes : Carnets de lectures

Mots-clés : nature, paysage

Keywords : Nature, Landscape

\section{AUTEURS}

\section{ETIENNE GRÉSILLON}

Université Paris-Diderot Laboratoire LADYSS

etienne.gresillon@univ-paris-diderot.fr

\section{BERTRAND SAJALOLI}

Université d'Orléans

Laboratoire EA 1210 CEDETE

bertrand.sajaloli@univ-orleans.fr 